\title{
HYPERBOLIC HEXAGONS AND ALGEBRAIC CURVES IN GENUS 3
}

\author{
A. AIGON AND R. SILHOL
}

\section{Introduction}

One of the consequences of the uniformization theorem of Koebe and Poincare is that any smooth complex algebraic curve $C$ of genus $g>1$ is conformally equivalent to $\mathbb{H} / G$, where $G \subset \mathrm{PSL}_{2}(\mathbb{R})$ is a Fuchsian group and is naturally endowed with a hyperbolic metric. Conversely, any compact hyperbolic surface is isomorphic to an algebraic curve. Hence any curve of genus $g>1$ may be described in two ways, either by an equation or by a Fuchsian group.

Going from the hyperbolic metric to a Fuchsian group is feasible in general (see for example [7]). On the other hand going from the hyperbolic metric or the Fuchsian group to equations of the algebraic curve is still a largely unsolved problem (but see [5]). This is the problem we want to address in genus 3. A partial solution for genus 2, and also for so-called real hyperelliptic M-curves, was proposed in [3]. We first reformulate some of the results of [3] in terms of modular invariants of rectangular hyperbolic pentagons (modular invariants are a straightforward generalization of the modulus of a quadrangle). In particular we show that starting with a rectangular pentagon, we can in a very simple way express equations for ten algebraic curves of genus 2 (in general non-isomorphic) tiled by copies of such a pentagon, each curve corresponding to the choice of a pair of points among the five vertices of the pentagon.

We generalize to genus 3 curves tiled by copies of a rectangular hexagon. The generalization we obtain yields a completely automatic way to obtain equations (at least numerically) of 30 genus 3 curves (in general non-isomorphic) tiled by a given hexagon. This time the curves are differentiated by either choosing two points among the six vertices of the hexagon (this yields 15 hyperelliptic curves) or by decomposing the six vertices into three pairs (this yields 15 other curves, in general non-hyperelliptic).

One of the results of our method is that it leads to a fast and fairly efficient method to test general ideas on uniformization (fast and efficient because in the average case it only takes a few seconds, 5 to 10 , to obtain the 30 equations with an accuracy of about 13 or 14 digits).

Another result is that we can explicitly exhibit one or two parameter families of curves which are algebraic with respect to the natural projective structure of the moduli space and which at the same time are defined by algebraic relations between the lengths of geodesics (or the cosh of these) defining the hyperbolic structure of the surface. We can also exhibit explicit relations between such families. For

Received 31 May 2001; revised 4 January 2002.

2000 Mathematics Subject Classification 30F10, 14H15, 32G15. 
example we show in Subsection 7.5 that curves with equations $y^{2}-x^{8}+\alpha x^{4}-1=0$, $\alpha$ real and $-2<\alpha<2$, and equation $y^{4}-x^{4}+\alpha x^{2} z^{2}-z^{4}=0$ (the same $\alpha$ ) are obtained from the same fundamental domain with different (explicitly described) identification patterns (see equations (7.1) and (7.2)).

\section{The modular invariants of a polygon}

By a polygon we mean a compact simply connected plane domain with piecewise $C^{1}$ boundary. In practice the polygons we will consider will be hyperbolic polygons with piecewise geodesic boundary components but this is not needed for the moment.

Let $P$ be a polygon and let $a_{1}, a_{2}, a_{3}, a_{4}$ be four distinct points in cyclic order on the boundary of $P$. Then there is a unique conformal map $f$ that maps the interior of $P$ onto the upper half plane and is such that continuous extension of $f$ to the boundary of $P$ maps $a_{1}$ to $0, a_{2}$ to 1 and $a_{4}$ to $\infty$. Let

$$
\lambda\left(\left\{P,\left(a_{1}, a_{2}, a_{3}, a_{4}\right)\right\}\right)=f\left(a_{3}\right) .
$$

$\lambda\left(\left\{P,\left(a_{1}, a_{2}, a_{3}, a_{4}\right)\right\}\right)$ is by construction a conformal invariant and we will say that it is the modular invariant or the modulus of $\left\{P,\left(a_{1}, \ldots, a_{4}\right)\right\}$.

It is well known how to compute these invariants (see for example [6, Section 16.11]). An explicit and efficient method to compute these numerically is described in [3, Sections 3 and 4]. We sketch briefly here the formal results underlying this method and refer to [3] for more detail on the computational aspects.

Lemma 1.1. Let $h$ be the harmonic function on the interior of $P$ whose continuous extension to the boundary is 0 between $a_{4}$ and $a_{1}$ and 1 between $a_{2}$ and $a_{3}$, and that has zero derivative with respect to the unit outward-pointing vector field orthogonal to the boundary of $P$ on the remaining components of the boundary. Let

$$
\tau=\int_{P}\|\operatorname{Grad}(h)\|^{2} d s
$$

be the capacity of $h$ on $P$. Then

$$
\lambda\left(\left\{P,\left(a_{1}, a_{2}, a_{3}, a_{4}\right)\right\}\right)=\frac{\exp (\pi \tau)}{16} \prod_{n=1}^{\infty} \frac{(1+\exp (-(2 n-1) \pi \tau))^{8}}{(1+\exp (-2 n \pi \tau))^{8}} .
$$

Proof. Let $\lambda=\lambda\left(\left\{P,\left(a_{1}, a_{2}, a_{3}, a_{4}\right)\right\}\right)$ and note that by definition $\lambda>1$. There exist a unique rectangle in the complex plane, with vertices $0,1,1+i \tau$ and $i \tau$, and a unique conformal map $\varphi$ of this rectangle onto $\mathbb{H}$ such that 0 is mapped to 0,1 to $1,1+i \tau$ to $\lambda$ and $i \tau$ to $\infty$. Such a $\tau$ can easily be computed using an elliptic integral and conversely the formula in the lemma expresses $\lambda$ in terms of $\tau$ (see for example $[8])$.

Let $f$ be the conformal map of the polygon onto $\mathbb{H}$ defining the modular invariant. Then, $\varphi^{-1} \circ f$ is a conformal map of the polygon onto the rectangle mapping $a_{1}$ to $0, a_{2}$ to 1 and so forth. Let $h=\mathfrak{R e}\left(\varphi^{-1} \circ f\right)$. This is a harmonic function on the polygon and its boundary behaviour is easily seen to be the one described in the lemma. However the capacity is a conformal invariant (see for example [6, 16.11f]); hence

$$
\int_{P}\|\operatorname{Grad}(h)\|^{2} d s=\int_{R}\|\operatorname{Grad}(\Re e(z))\|^{2} d x d y=\tau,
$$

and this proves the lemma. 


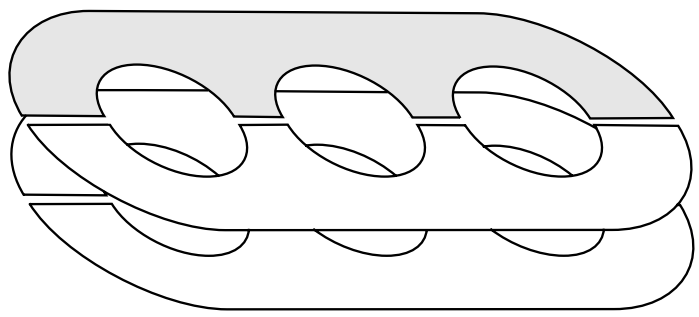

FigURE 1.

An illustration of the importance of these modular invariants is given in the next lemma, but first we need a construction.

Let $P$ be a hyperbolic polygon with $n$ geodesic boundary components $(n \geqslant 4)$. Let $g \leqslant(n-2) / 2$ and choose $2 g+2$ distinct vertices of the boundary, $a_{1}, \ldots, a_{2 g+2}$, in cyclic order. Let $\Sigma$ be the surface obtained by assembling four copies of $P$, two direct images and two mirror images, as in Figure 1 (where the identified edges are the $\left.\left[a_{i}, a_{i+1}\right]\right)$. This surface $\Sigma$ has a unique conformal structure compatible with its hyperbolic structure, and can hence also be considered as a Riemann surface or otherwise an algebraic curve $C$.

Lemma 1.2. An equation for the curve $C$ constructed above from four copies of $P$ is

$$
y^{2}=x(x-1)\left(x-\lambda_{1}\right) \ldots\left(x-\lambda_{2 g-1}\right)
$$

where $\lambda_{i}=\lambda\left(\left\{P,\left(a_{1}, a_{2}, a_{i+2}, a_{2 g+2}\right)\right\}\right)$.

Proof. We first note that by construction $\Sigma$ is of genus $g$ and has two anticonformal involutions, exchanging $P$ and its mirror images. Hence $C$ is a real curve with at least two real structures both with $g+1$ real components. Moreover the composition of these two anti-conformal involutions is a conformal involution with $2 g+2$ fixed points, the $a_{i}$. Since the genus is $g$ this means that $C$ is hyperelliptic. In particular $C$ has an equation of the form $y^{2}=x(x-1)\left(x-\mu_{1}\right) \ldots\left(x-\mu_{2 g-1}\right)$, with $\mu_{i} \in \mathbb{R}, 1<\mu_{i}<\mu_{i+1}$. By construction there is a branched two-to-one map from $\Sigma$ to $\mathbb{P}^{1}(\mathbb{C})$ that maps conformally the interior of $P$ onto the upper half plane and the points $a_{1}, \ldots, a_{2 g+2}$ to $0,1, \mu_{1}, \ldots, \mu_{2 g-1}, \infty$. From the uniqueness of such a map and the uniqueness of the maps defining the modular invariants, we conclude that for all $i, \lambda_{i}=\mu_{i}$; hence the lemma.

REMARK 1.3. (1) Lemma 1.2 is a mild improvement on [3, (3.8)] in the sense that it avoids the resort to theta characteristics and in applications simplifies the computations.

(2) We insist here again that, for small values of $n$ (and tested up to $n=8$, but there are also some restrictions on the values of the angles, and on the lengths, which should not be too large or too small), the methods indicated in [3] lead to a fast and efficient way of computing a numerical approximation of the modular invariants of an $n$-gon.

(3) For an $n$-gon $P$ with vertices $a_{1}, \ldots, a_{n}$ the various modular invariants one can associate to $P$ and a choice of four points are not all independent and in fact depend only on $n-3$ of these. This easily follows from the fact that a conformal automorphism of the upper-half plane fixing three points is the identity. 
(4) Let $P$ be a hyperbolic polygon with modular invariants $\lambda_{1}, \ldots, \lambda_{n}$ and let $s$ be the edge mapped, by the conformal map defining the modular invariants, onto $[\infty, 0]$. Let $P^{\prime}$ be the mirror image of $P$ and $s^{\prime}$ be the image of $s$ in $P^{\prime}$. Let $Q$ be the polygon obtained by assembling $P$ and $P^{\prime}$ along $s$ and $s^{\prime}$. Then, by the Schwarz reflection lemma, there is a conformal map from $Q$ onto the upper half plane $\mathbb{H}$ sending the vertices of $Q$ to $-\sqrt{\lambda_{n}}, \ldots,-\sqrt{\lambda_{1}},-1,1, \sqrt{\lambda_{1}}, \ldots, \sqrt{\lambda_{n}}$.

\section{Right-angled pentagons and genus 2 curves}

The results of this section are essentially a reformulation of those of [3], but we are going to use this relatively simple case to introduce our general method and point out the simplifications introduced by considering the problem in terms of modular invariants.

We will deal here with genus 2 curves obtained by assembling eight isometric right-angled pentagons. The equations of all the curves obtained in this way will only depend on the modular invariants of the pentagon and can be recovered quite easily.

Start with a right-angled hyperbolic pentagon $P$ with vertices $a_{1}, \ldots, a_{5}$. As before, let $f$ be the conformal map of $P$ onto $\mathbb{H}$, mapping respectively $a_{1}, a_{2}, a_{3}, a_{4}$ and $a_{5}$ onto $0,1, \lambda, \lambda^{\prime}$ and $\infty$.

Assemble $P$ and a mirror image $P^{\prime}$ along the edge $\left[a_{5}, a_{1}\right]$ to obtain a hexagon $H$ (see Figure 2). The map $g=\sqrt{f}$ maps conformally $P$ onto the domain $\mathfrak{I} m(z)>0$, $\Re e(z)>0$ and, as in Remark 1.3(4), it can be extended to a map of $H$ onto the upper half plane $\mathbb{H}$ mapping the vertices $a_{2}^{\prime}$ and $a_{2}$ to -1 and $1, a_{3}^{\prime}$ and $a_{3}$ to $-\sqrt{\lambda}$ and $\sqrt{\lambda}, a_{4}^{\prime}$ and $a_{4}$ to $-\sqrt{\lambda^{\prime}}$ and $\sqrt{\lambda^{\prime}}$ (where the $a^{\prime}$ are the vertices of $P^{\prime}$ ).

The genus 2 curve $C$ built as in Lemma 1.2 from this hexagon has the equation

$$
y^{2}=\left(x^{2}-1\right)\left(x^{2}-\lambda\right)\left(x^{2}-\lambda^{\prime}\right) .
$$

If now $P$ and $P^{\prime}$ are assembled along the edge $\left[a_{4}, a_{5}\right]$ (in place of $\left[a_{5}, a_{1}\right]$ ) we obtain a new hexagon $H^{\prime}$ (see Figure 2) and a new curve $C^{\prime}$. The equation of $C^{\prime}$ can be recovered as follows.

Let $A: z \longmapsto \lambda^{\prime} /\left(\lambda^{\prime}-z\right)$, and let $f^{\prime}=A \circ f$. The map $f^{\prime}$ also maps $P$ onto the upper half plane but this time it maps the edge $\left[a_{4}, a_{5}\right]$ onto the negative real half line. Setting $g^{\prime}=\sqrt{f^{\prime}}, g^{\prime}$ maps $P$ onto the upper right-hand quarter plane and $\left[a_{4}, a_{5}\right]$ onto the upper half of the pure imaginary axis. Again we can extend this

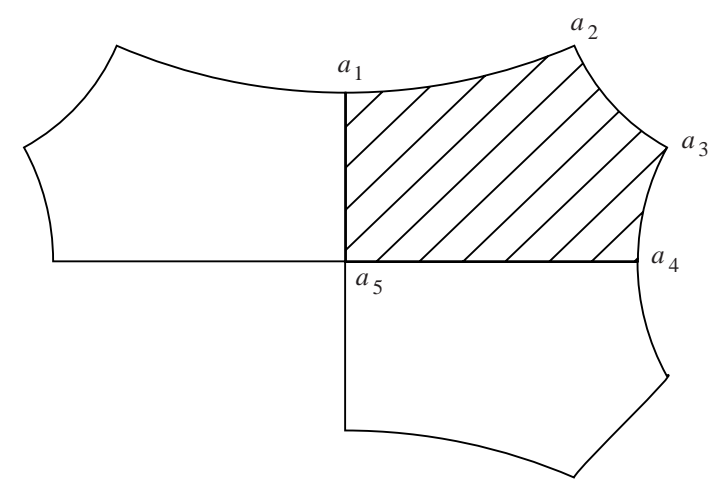

FigURE 2. 
map to a map from $H^{\prime}$ onto the upper half plane. This leads for $C^{\prime}$ to the equation

$$
y^{2}=\left(x^{2}-1\right)\left(x^{2}-\frac{\lambda^{\prime}}{\lambda^{\prime}-1}\right)\left(x^{2}-\frac{\lambda^{\prime}}{\lambda^{\prime}-\lambda}\right) .
$$

As there are five different ways to build a hexagon by assembling $P$ and a mirror image of $P$, we will obtain in this way five different curves (in general distinct) and their equations can be obtained just as simply as the preceding ones. These five curves correspond to those obtained with the $D_{5}$-action in [3].

However pentagons can also be assembled differently to obtain a genus 2 curve (see for example the construction in [3, Section 7]). To describe these other constructions we reconsider the preceding ones. The pull backs, in the algebraic curve $C$, of three of the vertices of the pentagon $P$ are Weierstrass points. The other two are not Weierstrass points but rather fixed points of non-hyperelliptic conformal automorphisms. Clearly we can in fact choose any pair of vertices of the pentagon to lift to non-Weierstrass points.

To obtain an equation for the corresponding algebraic curves we proceed exactly along the same lines as before. For example, for one of these we can use $A^{\prime}$ : $z \longmapsto z-1$ and set $f^{\prime \prime}=A^{\prime} \circ f$. Then $f^{\prime \prime}$ will map $P$ onto the upper half plane with $f^{\prime \prime}\left(a_{1}\right)=-1, f^{\prime \prime}\left(a_{2}\right)=0$ and $f^{\prime \prime}\left(a_{5}\right)=\infty$. Taking again $g^{\prime \prime}=\sqrt{f^{\prime \prime}}$ we obtain a map from the pentagon onto the upper right-hand quarter plane. Modifying in an obvious way the above construction we conclude that the algebraic curve $C^{\prime \prime}$ obtained in this way will have the equation

$$
y^{2}=\left(x^{2}+1\right)\left(x^{2}-\lambda+1\right)\left(x^{2}-\lambda^{\prime}+1\right) .
$$

In this way we can recover the constructions described in $[3$, Section 5 and Section 7].

To formulate more formally the results we have obtained, we consider the fundamental domain obtained by pasting eight copies of a generic rectangular pentagon, that is, one with no two edges of equal length (see Figure 3). It is readily checked that up to isometry or rotation of the pentagon there are only two possible side identifications to obtain a smooth genus 2 surface tiled by these pentagons (where by a tiling we mean one where one passes from a pentagon to an adjacent one by a reflection).

These are, using the notation of Figure 3, as follows:

(i) $a, b, c, d, e-h$ and $f-g$;

(ii) $a, b, c, d, e-g$ and $f-h$.

(Identifying one side labelled $c$ with one labelled $d$ would violate the above conditions.)

In both cases the hyperelliptic involution is induced by the central symmetry. On the other hand in case (i) the hyperelliptic involution fixes all the images of the vertices of the pentagon except for $p$ and $q$ while in case (ii) it fixes all the vertices except $p$ and $r$. We are exactly in the situation described previously; hence we have the following lemma.

LEMMa 2.1. Let $f$ be the conformal map from the pentagon onto the upper half plane mapping $p$ to $\infty, q$ to 0 and $r$ to 1 . Let $\lambda$ and $\mu$ be the images of the other two vertices.

Then an equation for the algebraic curve obtained using identification pattern (i) above is 


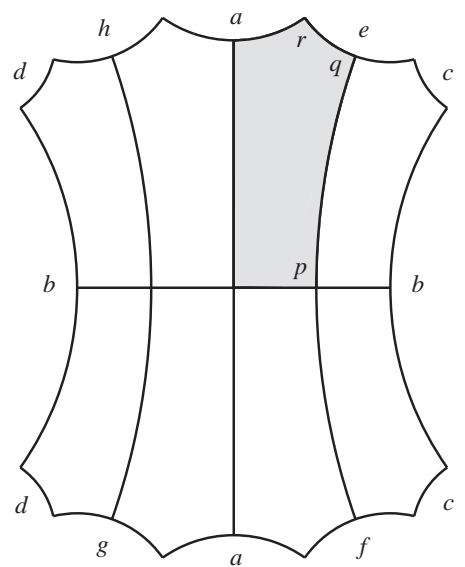

FIGURE 3 .

$1: y^{2}=\left(x^{2}-1\right)\left(x^{2}-\lambda\right)\left(x^{2}-\mu\right)$

An equation for the curve obtained by using identification pattern (ii) is $6: y^{2}=\left(x^{2}+1\right)\left(x^{2}-(\lambda-1)\right)\left(x^{2}-(\mu-1)\right)$.

Moreover by rotating the pentagon we obtain the curves with equations

$2: y^{2}=\left(x^{2}-1\right)\left(x^{2}-\frac{\mu}{\mu-1}\right)\left(x^{2}-\frac{\mu}{\mu-\lambda}\right)$

$3: y^{2}=\left(x^{2}-1\right)\left(x^{2}-\frac{\mu}{\lambda}\right)\left(x^{2}-\frac{\mu-1}{\lambda-1}\right)$

$4: y^{2}=\left(x^{2}-1\right)\left(x^{2}-\frac{\mu-1}{\mu-\lambda}\right)\left(x^{2}-\frac{(\mu-1) \lambda}{\mu-\lambda}\right)$

$5: y^{2}=\left(x^{2}-1\right)\left(x^{2}-\frac{(\mu-1) \lambda}{\mu(\lambda-1)}\right)\left(x^{2}-\frac{\lambda}{\lambda-1}\right)$

for identification pattern (i), and those with equations

$$
\begin{aligned}
& 7: y^{2}=\left(x^{2}+1\right)\left(x^{2}-\frac{1}{\lambda-1}\right)\left(x^{2}-\frac{\lambda}{\mu-\lambda}\right) \\
& 8: y^{2}=\left(x^{2}+1\right)\left(x^{2}-\frac{\mu-\lambda}{\lambda}\right)\left(x^{2}-\frac{\mu-\lambda}{\lambda-1}\right) \\
& 9: y^{2}=\left(x^{2}+1\right)\left(x^{2}-\frac{\lambda-1}{\mu-\lambda}\right)\left(x^{2}-\frac{(\lambda-1) \mu}{\mu-\lambda}\right) \\
& 10: y^{2}=\left(x^{2}+1\right)\left(x^{2}-\frac{\mu-\lambda}{\mu(\lambda-1)}\right)\left(x^{2}-\frac{1}{\lambda-1}\right)
\end{aligned}
$$

for identification pattern (ii).

Considering pentagons that yield two or more isometric surfaces leads to some of the special families and special curves described in [3]. The results of this section can also be generalized in another direction, by considering covers of the sphere minus 5 points. This is done in [1]. 


\section{Genus 3 double covers of a torus}

In the following sections we are going to generalize to genus 3 curves using hexagons in place of pentagons. However, the situation is more involved, since in genus 3 not all curves are hyperelliptic and we will need new tools. The first of these is an explicit way to obtain equations for genus 3 double covers of a fixed genus 1 curve ramified over four given distinct points.

Proposition 3.1. Let $C$ be a genus 1 curve, and let $p_{1}, p_{2}, p_{3}, p_{4}$ be four distinct points on $C$. Then there exist at most four distinct non-isomorphic genus 3 double covers of $C$ ramified over the $p_{i}$. Moreover each of these can be distinguished by a specific choice of an origin for the elliptic group structure on $C$ and the equations of these double covers can be explicitly recovered in terms of such a choice.

Proof. It is well known that there exist 16 points $p$ on $C$ such that the divisors $\Sigma p_{i}$ and $4 p$ are linearly equivalent (see for example [4] or [11]). Clearly if $p$ and $p^{\prime}$ are two choices then $p-p^{\prime}$ is of order 4 in $\operatorname{Pic}^{0}(C)$. We choose such a $p$.

Step 1: This is the construction of a covering $C_{p}$ with $p$ such that $\Sigma p_{i} \sim 4 p$ (linear equivalence).

Let $(C, p)$ be the elliptic curve with origin at $p$, and $(x, y)$ be Weierstrass coordinate functions on $(C, p)$, that is, such that $(x, y, 1)$ is a basis of $\mathscr{L}(3 p)$ providing for $C$ an affine equation of the form

$$
\left(E_{p}\right) \quad y^{2}=4 x^{3}-g_{2} x-g_{3}=4\left(x-a_{1}\right)\left(x-a_{2}\right)\left(x+a_{1}+a_{2}\right) .
$$

In place of the preceding embedding we can also consider that of $C$ in $\mathbb{P}^{3}$ associated to the basis $\left(x, y, x^{2}, 1\right)$ of $\mathscr{L}(4 p)$ which maps $C$ onto $\left\{[x, y, t, z] \in \mathbb{P}^{3} / x^{2}=\right.$ $\left.t z, y^{2}=4 x t-g_{2} x z-g_{3} z^{2}\right\}$. In this case the image of the divisor $\Sigma p_{i}$ will be defined by a hyperplane section $a x+b y+c z+d t=0$.

If $b=0$, this means that the coordinates of the $p_{i}$ associated to equation (3.1) are of the form $\left(x_{1}, \pm y_{1}\right),\left(x_{2}, \pm y_{2}\right)$. Transforming this equation via $x \longmapsto\left(x-x_{1}\right) /\left(x-x_{2}\right)$, we get an equation for $C$ of the form $y^{2}=(x-1)\left(x-b_{1}\right)\left(x-b_{2}\right)\left(x-b_{3}\right)$, where the points $p_{i}$ are over $x=0$ and $x=\infty$. In this case we define the genus 3 curve $C_{p}$ to be the one defined by the equation $y^{2}=\left(x^{2}-1\right)\left(x^{2}-b_{1}\right)\left(x^{2}-b_{2}\right)\left(x^{2}-b_{3}\right)$. This is a double cover of $C$ branched at the $p_{i}$.

If $b \neq 0$, we may assume that $b=1$. The transformation of $\mathbb{P}^{3}$ given by $[x, y, t, z] \longmapsto$ $[x, y-a x-c t-d z, t, z]$ enables us to realize $C$ in $\mathbb{P}^{3}$ as $\left(E_{p}\right)$,

$$
\left\{[x, y, t, z] \in \mathbb{P}^{3} / x^{2}=t z, y^{2}+2 y \tilde{f}_{1}(x, t, z)+\tilde{f}_{0}(x, t, z)=0\right\},
$$

with $\tilde{f}_{1}$ homogeneous of degree 1 and $\tilde{f}_{0}$ homogeneous of degree 2 . The $p_{i}$ are now the points of intersection with the hyperplane $\{y=0\}$. Set $f_{1}(x)=\tilde{f}_{1}\left(x, x^{2}, 1\right)$ and $f_{0}(x)=\tilde{f}_{0}\left(x, x^{2}, 1\right)$, then $y^{2}+2 y f_{1}(x)+f_{0}(x)=0$ is an affine equation for $C$. In this case we define $C_{p}$ to be the curve defined by the affine equation $y^{4}+2 y^{2} f_{1}(x)+f_{0}(x)=$ 0 . It is by construction a double cover of $C$ branched at the $p_{i}$.

The coverings $C_{p}$ we have constructed do not depend on the choice of Weierstrass coordinate functions for $(C, p)$. If $(x, y)$ is one choice of Weierstrass coordinate functions for $(C, p)$ then all other pairs will be of the form $\left(\lambda^{2} x, \lambda^{3} y\right)$ and from this it is readily shown that the construction does not depend on such a choice.

Step 2: If $\tilde{C}$ is a double cover of $C$ branched at the $p_{i}$, then $\tilde{C}$ is isomorphic to $C_{p}$ for some $p$ such that $4 p \sim \Sigma p_{i}$.

As before we will treat separately the hyperelliptic case. 
If $\tilde{C}$ is hyperelliptic, the involution $\varphi$ such that $C \simeq \tilde{C} / \varphi$ can be normalized as $(x, y) \longmapsto(-x, y)$. The equation of $\tilde{C}$ will then be of the form $y^{2}=\left(x^{2}-b_{1}\right)\left(x^{2}-\right.$ $\left.b_{2}\right)\left(x^{2}-b_{3}\right)\left(x^{2}-b_{4}\right)$ and this induces the equation $y^{2}=\left(x-b_{1}\right)\left(x-b_{2}\right)\left(x-b_{3}\right)\left(x-b_{4}\right)$ for $C$, the $p_{i}$ being over $x=0$ and $x=\infty$.

Let $r_{j}=\left(b_{j}, 0\right)$. We then have obviously, for all $j, \Sigma p_{i} \sim 4 r_{j}$ (note that the $r_{i}$ define points of order 2 in $C_{r_{j}}$ ). From the construction of $C_{r_{j}}$ it is then easy to construct, by means of a suitable Möbius transformation, an isomorphism between $\tilde{C}$ and $C_{r_{j}}$. This proves the assertion for hyperelliptic double covers.

If $\tilde{C}$ is not hyperelliptic, we can realize it as a smooth quartic in $\mathbb{P}^{2}$. Since moreover this realizes $C$ as a canonical curve the involution $\varphi$ can be normalized as $(x, y) \longmapsto(x,-y)$. Hence we can choose $\tilde{C}$ to have an equation of the form $y^{4}+2 y^{2} f_{1}(x)+f_{0}(x)=0$ and hence the quotient $C$ to have the equation

$$
y^{2}+2 y f_{1}(x)+f_{0}(x)=0,
$$

the $p_{i}$ being defined by $f_{0}(x)=0, y=0$. Let $r_{j}, j=1, \ldots, 4$, be the points defined by $y+f_{1}(x)=0$. The map $(x, y) \longmapsto x$ is two-to-one and precisely branched at the $r_{j}$. This means again that if we choose one of the $r_{j}$ to be the origin of the group structure on $C$ the others will be points of order 2 .

We map $C$ into $\mathbb{P}^{3}$ via the map $\psi:(x, y) \longmapsto\left(x, y, x^{2}, 1\right)$. The $r_{j}$ are now defined by the hyperplane section $\left\{y+\tilde{f}_{1}(x, t, z)=0\right\}$, where $\tilde{f}_{1}(x, t, z)$ is the homogeneous polynomial of degree 1 such that $\tilde{f}_{1}\left(x, x^{2}, 1\right)=f_{1}(x)$. Since the $p_{i}$ are also defined by a hyperplane section, $\{y=0\}$, we have $\Sigma p_{i} \sim \Sigma r_{j}$. However, as remarked above, the $r_{j}$ can be chosen to form the subgroup of points of order 2; hence $\Sigma r_{j} \sim 4 r_{k}$ and hence, for any choice of $j, \Sigma p_{i} \sim 4 r_{j}$.

We are going to show, as in the hyperelliptic case, that $\tilde{C}$ is in fact isomorphic to $C_{r_{j}}$, for any and hence all $r_{j}, j=1, \ldots, 4$.

Let $g: x \longmapsto(\alpha x+\beta) /(\gamma x+\delta), \alpha \delta-\beta \gamma=1$ be a Möbius transformation yielding for $C$ the Weierstrass equation $v^{2}=4 u^{3}-g_{2} u-g_{3}$ and mapping $r_{j}$ to infinity.

Let $v$ be the leading coefficient of $f_{1}^{2}-f_{0}$, and set

$$
\eta=\sqrt{\left(-\gamma^{4} g_{3}-g_{2} \gamma^{3} \delta+4 \gamma \delta^{3}\right) / v}
$$

if $\gamma \neq 0, \eta=2 / \sqrt{v}$ otherwise. Then one can check that the map

$$
(x, y) \longmapsto\left(\frac{\alpha x+\beta}{\gamma x+\delta}, \sqrt{\eta} \frac{y}{\gamma x+\delta}\right)
$$

induces an isomorphism between $\tilde{C}$ and $C_{r_{j}}$.

REMARK 3.2. (1) Note that as a consequence of the above proof we have proved that if $p-p^{\prime}$ is of order 2 in $\operatorname{Pic}^{0}(C)$ then $C_{p}$ and $C_{p^{\prime}}$ are isomorphic.

Since the subgroups of points of order 4 and 2 in $\operatorname{Pic}^{0}(C)$ are of respective orders 16 and 4, we have also proved that there are at most four non-isomorphic $C_{p}$.

In Sections 4 and 5 we will show that in general the four covers we have built are not isomorphic.

(2) Let $C$ be a genus 1 curve defined by an affine equation of the form $y^{2}=P(x)$ with $\operatorname{deg}(P)=3$ or 4 . One can embed $C$ in $\mathbb{P}^{3}$ via $(x, y) \longmapsto\left[x, y, x^{2}, 1\right]$. Select a hyperplane section defining four points $p_{i}, i=1, \ldots, 4$, on this embedded curve. From this situation one can use exactly the same technique as in Proposition 3.1 to build a genus 3 double cover ramified over the $p_{i}$. Let $p$ be one of the four points 
of affine coordinates $\left(x_{i}, 0\right)$, with $P\left(x_{i}\right)=0$. According to (3.1), this double cover is isomorphic to $C_{p}$. We summarize this as follows.

A genus 3 double cover of $C$ is completely described by

(i) an affine equation $y^{2}=P(x)$ with $\operatorname{deg} P=3$ or 4 for $C$; and

(ii) an equation of a plane $a x+b y+c t+d z=0$ in $\mathbb{P}^{3}$.

For further use we will need a slightly different description of the double covers. For this we introduce the following notation.

$C_{p}\left(\left\{p_{1}, p_{2}, p_{3}, p_{4}\right\}\right)$ will be the double cover of $C$ branched over the $p_{i}$ and defined by the choice of $p$, with $4 p \sim \Sigma p_{i}$ as in the proof of Proposition 3.1.

With this we have the following lemma.

Lemma 3.3. Let $C$ be of genus 1 and $p_{1}, \ldots, p_{4}$ be four points on $C$. Let $p$ be such that $4 p \sim \Sigma p_{i}$. Let $q \in C$ be a point defining an order 4 element of the elliptic curve $(C, p)$ and let $t_{q}$ be the translation by this element. Then

$$
C_{q}\left(\left\{p_{1}, p_{2}, p_{3}, p_{4}\right\}\right) \cong C_{p}\left(\left\{t_{q}\left(p_{1}\right), t_{q}\left(p_{2}\right), t_{q}\left(p_{3}\right), t_{q}\left(p_{4}\right)\right\}\right) .
$$

Proof. We first note that since by definition $4(q-p) \sim 0$ we have $\Sigma p_{i} \sim 4 q$ and $\Sigma t_{q}\left(p_{i}\right) \sim \Sigma p_{i} \sim 4 p$. This shows that the genus 3 coverings are well defined. Second, if $q^{\prime}$ is such that $t_{q}\left(q^{\prime}\right)=p$, then $q^{\prime}$ defines the inverse of $q$ in $(C, p)$ and hence $2\left(q-q^{\prime}\right) \sim 4(q-p) \sim 0$. By Remark 3.2(1), this means that $C_{q}\left(\left\{p_{1}, p_{2}, p_{3}, p_{4}\right\}\right) \cong$ $C_{q^{\prime}}\left(\left\{p_{1}, p_{2}, p_{3}, p_{4}\right\}\right)$.

Now $t_{q}$ is an automorphism of $C$ sending $q^{\prime}$ to $p$ and the $p_{i}$ to the $t_{q}\left(p_{i}\right)$. Hence, $C_{q^{\prime}}\left(\left\{p_{1}, p_{2}, p_{3}, p_{4}\right\}\right) \cong C_{p}\left(\left\{t_{q}\left(p_{1}\right), t_{q}\left(p_{2}\right), t_{q}\left(p_{3}\right), t_{q}\left(p_{4}\right)\right\}\right)$.

COROLlary 3.4. Let $q_{0} \in C$ be such that $\Sigma p_{i} \sim 4 q_{0}$. Let $q_{1}, q_{2}$ and $q_{3}$ be points of order 4 on the elliptic curve $\left(C, q_{0}\right)$ such that $q_{i}-q_{j}, 0 \leqslant i<j \leqslant 3$, is not of order 2 . Then the four possibly distinct genus 3 double covers of $C$ branched at the $p_{i}$ are the $C_{q_{0}}\left(\left\{t_{j}\left(p_{1}\right), \ldots, t_{j}\left(p_{4}\right)\right\}\right)$, where we have written $t_{j}$ for $t_{q_{j}}$.

\section{Right-angled hexagons and genus 3 surfaces}

We start by assembling four copies of a hexagon as in Figure 4.

\section{1.}

It is readily checked that up to isometry or rotation of the hexagon there are only three possible side identifications to obtain a genus 1 surface with four cone points (each with total angle $\pi$ ) and tiled by the hexagons (again where one passes from a hexagon to an adjacent one by a reflection). These are, using the notation of Figure 4, as follows:

(i) $1,7,4,2-11,3-5,6-8$ and $9-10$;

(ii) $1,7,4,2-6,3-5,8-11$ and $9-10$;

(iii) $1,7,4,2-6,3-10,5-9$ and $8-11$.

In each case the symmetry centred at $p$ induces an isometry $\varphi$ on the surface. In case (i) the other fixed points of $\varphi$ are $q, r$ and $s$ (and their images); in case (ii) the other fixed points are $q, r$ and $t$ and in case (iii) $q, s$ and $t$. The cone points are also 


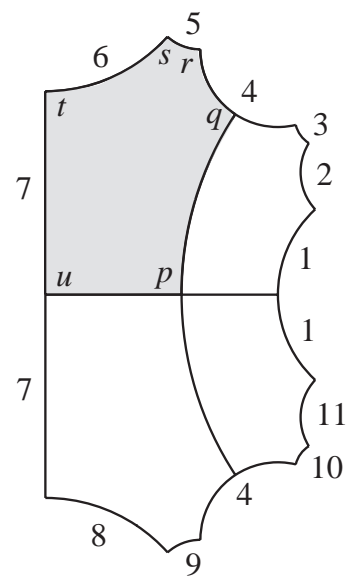

Figure 4.

easy to identify. They are above $u$ and $t$ in case (i), above $u$ and $s$ in case (ii) and above $u$ and $r$ in case (iii).

From this it is easy, using the method of Section 1, to obtain an equation of the genus 1 curve and the coordinates of the marked points. Let $f$ be the conformal map that maps the hexagon onto the upper half plane, the point $p$ to $\infty$, the point $q$ to 0 and the point $r$ to 1 . Let $f(s)=\lambda, f(t)=\mu$ and $f(u)=v$. The genus 1 curve will have the following equation.

\section{2.}

(i) $y^{2}-x(x-1)(x-\lambda)$ in case (i) and the cone points will be above $x=\mu$ and $x=v$.

(ii) $y^{2}-x(x-1)(x-\mu)$ in case (ii) and the cone points will be above $x=\lambda$ and $x=v$.

(iii) $y^{2}-x(x-1)(x-\mu / \lambda)$ in case (iii) and the cone points will be over $x=1 / \lambda$ and $x=v / \lambda$.

Following the results of Section 3 there are in each case four distinct genus 3 double covers branched respectively over the points $u$ and $t, u$ and $s$, and $u$ and $r$. To describe these double covers we introduce the fundamental domain obtained by doubling that of Figure 4 (see Figure 5). On this domain we consider the identification patterns yielding a smooth genus 3 surface, tiled by the hexagons and such that the central symmetry induces a double covering of one of the genus 1 surfaces just described and precisely branched at the cone points. We will consider the following list (we will show below that for a generic hexagon no two of these surfaces are isometric, and that hence this is a complete list).

(1.i) $1,2-21,3-5,4-19,6-17,7-16,8-10,9-14,11-12,13-15,18-20$;

(1.ii) $1,2-16,3-5,4-19,6-12,7-21,8-10,9-14,11-17,13-15,18-20$;

(1.iii) $1,2-16,3-10,4-19,5-8,6-12,7-21,9-14,11-17,13-20,15-18$; 


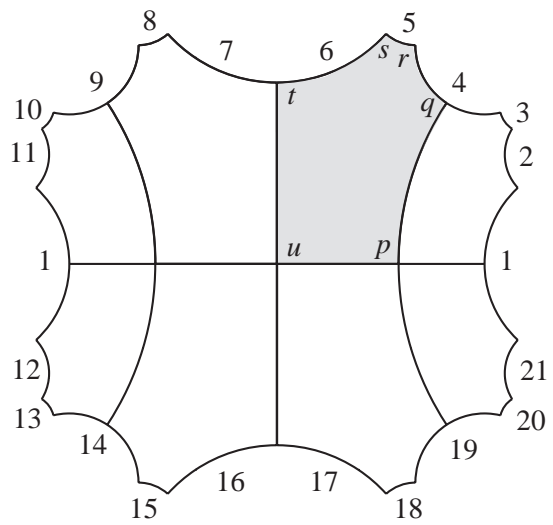

FiguRE 5.

(2.i) $1,2-21,3-5,4-9,6-17,7-16,8-10,11-12,13-15,14-19,18-20$;

(2.ii) $1,2-16,3-5,4-9,6-12,7-21,8-10,11-17,13-15,14-19,18-20$;

(2.iii) 1, 2-16, 3-20, 4-9, 5-18, 6-12, 7-21, 8-15, 10-13, 11-17, 14-19;

(3.i) $1,2-21,3-15,4-19,5-13,6-17,7-16,8-20,9-14,10-18,11-12$;

(3.ii) $1,2-6,3-15,4-19,5-13,7-11,8-20,9-14,10-18,12-16,17-21$;

(3.iii) 1, 2-6, 3-10, 4-19, 5-8, 7-11, 9-14, 12-16, 13-20, 15-18, 17-21;

(4.i) $1,2-21,3-15,4-9,5-13,6-17,7-16,8-20,10-18,11-12,14-19$;

(4.ii) $1,2-6,3-15,4-9,5-13,7-11,8-20,10-18,12-16,14-19,17-21$;

(4.iii) 1, 2-6, 3-20, 4-9, 5-18, 7-11, 8-15, 10-13, 12-16, 14-19, 17-21.

\section{4.}

To distinguish more simply each case we consider how the isometry $\varphi$ in Subsection 4.1 lifts to these genus 3 surfaces. We easily find the following lemma.

Lemma 4.1. The isometry $\varphi$ of the genus 1 quotient described in Subsection 4.1 lifts in each case to two conformal involutions $\psi_{1}$ and $\psi_{2}$ and the composition $\psi_{0}=\psi_{1} \circ \psi_{2}$ is the involution induced by the central symmetry in Figure 5.

In cases (1.i), (1.ii) and (1.iii) in Subsection 4.3, one of $\psi_{1}$ and $\psi_{2}$ has eight fixed points while the other has none. In particular the genus 3 surface is always hyperelliptic in these cases.

In all other cases the two involutions $\psi_{1}$ and $\psi_{2}$ each have four fixed points. For $i=0,1$ or $2 \psi_{i}$ fixes exactly two vertices of the hexagons. We can describe this by giving a set of three pairs, the first corresponding to the vertices fixed by $\psi_{0}$, the second to those fixed by $\psi_{1}$ and the last to those fixed by $\psi_{2}$. With this notation we have the following:

(1) $(u, t),(p, s),(q, r)$ for case (2.i);

(2) $(u, s),(p, t),(q, r)$ for case (2.ii);

(3) $(u, r),(p, t),(q, s)$ for case (2.iii);

(4) $(u, t),(p, q),(r, s)$ for case (3.i);

(5) $(u, s),(p, q),(r, t)$ for case (3.ii);

(6) $(u, r),(p, q),(s, t)$ for case (3.iii); 
(7) $(u, t),(p, r),(q, s)$ for case (4.i);

(8) $(u, s),(p, r),(q, t)$ for case (4.ii);

(9) $(u, r),(p, s),(q, t)$ for case (4.iii).

As an immediate consequence of Lemma 4.1 we note the following.

Lemma 4.2. For a generic rectangular hexagon (that is, one such that no two edges have the same length), no two surfaces in the list in Subsection 4.3 are isometric.

Proof. For a generic hexagon the only automorphisms of the surface will be those described in Lemma 4.1. In cases (1.i), (1.ii) and (1.iii) there will be only one genus 1 quotient and these will have respectively the equations in (i), (ii) and (iii) in Subsection 4.2 which correspond to non-isomorphic curves in the generic case.

In each other case we will have three distinct genus 1 quotients. Equations for these quotients are easy to express in terms of the modular invariants of the hexagon. For example using the notations of Subsection 4.2, the quotients will have the following equations:

(1) $y^{2}=x(x-1)(x-\lambda), y^{2}=x(x-1)(x-\mu)(x-v)$ and $y^{2}=(x-\lambda)(x-\mu)(x-v)$ in case (2.i);

(2) $y^{2}=x(x-1)(x-\mu), y^{2}=x(x-1)(x-\lambda)(x-v)$ and $y^{2}=(x-\lambda)(x-\mu)(x-v)$ in case (2.ii);

and so forth. From this we can again conclude that for a generic hexagon the surfaces are non-isometric.

Allowing for rotations of the hexagon, we can apply Lemmas 4.1 and 4.2 to prove the following proposition.

Proposition 4.3. Starting from a rectangular hexagon the construction described in Subsection 4.3 yields 30 genus 3 surfaces. For a generic hexagon these 30 surfaces will all be distinct and exactly 15 will be hyperelliptic. The 15 hyperelliptic surfaces can be distinguished by the choice of two vertices among six, and the 15 others by the choice of a decomposition of the six vertices of the hexagon into three pairs.

Proof. This is just a reformulation of Lemma 4.1 taking into account the choice of the exact disposition of the hexagon in Figure 5. The proof of the assertion in the generic case follows exactly the same lines as the proof of Lemma 4.2.

\section{Equations for genus 3 surfaces built from right-angled hexagons}

We are going to use the results of Section 3 to give equations for the algebraic curves corresponding to the surfaces described in Section 4.

Since for cases (1.i)-(1.iii) the curves are hyperelliptic, the equations are easy to obtain. We have, with the notations of Subsection 4.2:

$$
\begin{gathered}
y^{2}-\left(x^{2}-1\right)\left(x^{2}-\frac{v}{\mu}\right)\left(x^{2}-\frac{v-1}{\mu-1}\right)\left(x^{2}-\frac{v-\lambda}{\mu-\lambda}\right) \\
y^{2}-\left(x^{2}-1\right)\left(x^{2}-\frac{v}{\lambda}\right)\left(x^{2}-\frac{v-1}{\lambda-1}\right)\left(x^{2}+\frac{v-\mu}{\mu-\lambda}\right) \\
y^{2}-\left(x^{2}-1\right)\left(x^{2}-v\right)\left(x^{2}+\frac{v-\lambda}{\lambda-1}\right)\left(x^{2}+\frac{v-\mu}{\mu-1}\right) .
\end{gathered}
$$


TABLE 1.

\begin{tabular}{|c|c|c|}
\hline & $\begin{array}{l}\text { Equation of } \\
\text { genus } 1 \text { curve }\end{array}$ & $\begin{array}{l}\text { Coefficients } \\
\text { of hyperplane }\end{array}$ \\
\hline (1) & $y^{2}=x(x-1)(x-\tau)$ & $a, b, c, d$ \\
\hline \multirow[t]{4}{*}{ (2) } & $y^{2}=\left(x^{2}-1\right)\left(x^{2}-\left(2 \tau-2 \sqrt{\tau^{2}-\tau}-1\right)^{2}\right)$ & $a_{2}=-2(\tau-1)(\sqrt{\tau}-\sqrt{\tau-1}) b$ \\
\hline & & $b_{2}=a+c+d / \tau$ \\
\hline & & $c_{2}=a+c+(2 \tau-1) d / \tau$ \\
\hline & & $d_{2}=\left(2 \sqrt{\tau^{2}-\tau}-2 \tau+1\right)(a+(2 \tau-1) c+d / \tau$ \\
\hline & $y^{2}=\left(x^{2}+1\right)\left(x^{2}+\left(\frac{1-\sqrt{\tau}}{1+\sqrt{\tau}}\right)^{2}\right)$ & $a_{3}=2 b \tau(\sqrt{\tau}-1)$ \\
\hline & & $b_{3}=-(\tau-1)(c \tau-d)$ \\
\hline & & $c_{3}=2 \tau a+(\tau+1)(c \tau+d)$ \\
\hline & & $d_{3}=(c \tau+d)(\sqrt{\tau}-1)^{2}$ \\
\hline \multirow[t]{4}{*}{ (4) } & $y^{2}=x^{4}-2(1-2 / \tau) x^{2}+1$ & $a_{4}=2 i(\tau-1) b / \sqrt{\tau}$ \\
\hline & & $b_{4}=-a-\tau c-d$ \\
\hline & & $c_{4}=a+\tau c-(\tau-2) d / \tau$ \\
\hline & & $d_{4}=a-(\tau-2) c+d$ \\
\hline
\end{tabular}

To give equations in the other cases we will also need a description of type $(*)$ for these first three cases. For this let $\tau$ be equal to $\lambda$ in case (i), $\mu$ in case (ii) and $\mu / \lambda$ in case (iii). Then the curve can be described by the equation $y^{2}=x(x-1)(x-\tau)$ for the genus 1 curve and the equations,

$$
\begin{aligned}
-(\mu+v) x+t+\mu v z & =0 \\
-(\lambda+v) x+t+\lambda v z & =0 \\
-(v+1) \lambda x+\lambda^{2} t+v z & =0
\end{aligned}
$$

for the hyperplane in $\mathbb{P}^{3}$.

Proposition 5.1. The surfaces in Subsection 4.3 can be defined as in (*) by an affine equation $y^{2}=P(x)$ and a hyperplane $a_{i} x+b_{i} y+c_{i} t+d_{i} z=0$, where the equation and $a_{i}, b_{i}, c_{i}, d_{i}$ are given in Table 1 , where $\tau$ is equal to $\lambda$ in case (i), to $\mu$ in case (ii) and to $\mu / \lambda$ in case (iii) and where the coefficients $a, b, c, d$ are those appearing in equations (5.2).

Moreover with the obvious labelling convention the genus 3 algebraic curve defined here will correspond to the surface with the same label in Subsection 4.3.

Proof. We fix $C$ as the genus 1 quotient; we want to consider its four points and write its equation in the form $y^{2}=x(x-1)(x-\tau)$. In the notations of Section 3 the four covers of $C$, branched over the given points, are then isomorphic to $C_{q_{j}}$, $j=0, \ldots, 3$, where the $q_{j}$ are some points of order 4 in $\left(C, q_{0}\right)$ such that $2\left(q_{j}-q_{k}\right) \neq 0$. Following Silverman [11, pp. 58-59] one can easily check that we can take for $q_{1}$, 
$q_{2}$ and $q_{3}$ the points of affine coordinates

$$
\begin{gathered}
q_{1}=\left(\tau+\sqrt{\tau^{2}-\tau}, \sqrt{\tau^{2}-\tau}(\sqrt{\tau-1}+\sqrt{\tau})\right), \\
q_{2}=(\sqrt{\tau}, i(\tau-\sqrt{\tau})), \\
q_{3}=q_{1}+q_{2}=(1-i \sqrt{\tau-1}, \tau-1+i \sqrt{\tau-1}),
\end{gathered}
$$

where $\tau$ is as in the proposition and depends on the choice of $C$ we have made.

Embed $C$ in $\mathbb{P}^{3}$ by applying $(x, y) \longmapsto\left[x, y, x^{2}, 1\right]$ to the above equation. The point $q_{0}$ is then defined by the hyperplane section $\{z=0\}$ and the $p_{i}$ by a hyperplane section $\{a x+b y+c t+d z=0\}$, where $a, b, c, d$ are the coefficients that appear in (5.2) and again depend on the case under consideration.

We are going to use Corollary 3.4 to describe the other coverings. For this we note that the points of order 4 of $\left(C, q_{0}\right)$ correspond to hyperplane sections intersecting the image of $C$ at single points (see for example [11]). The translations of order 4 are then projective transformations of $\mathbb{P}^{3}$ leaving globally the image of $C$ stable and exchanging two such hyperplanes. Moreover these transformations can be deduced from the group law algorithm given by Silverman [11, pp. 58-59].

Explicitly, if we write, as in Corollary 3.4, $t_{1}, t_{2}$ and $t_{3}$ for the translations of order 4 corresponding to the points $q_{1}, q_{2}$ and $q_{3}$, then the computations (following the method indicated in [11]) show that $t_{1}, t_{2}$ and $t_{3}$ are induced by the transformations of $\mathbb{P}^{3}$ defined by the matrices $A, B$ and $A B$, where

$$
\begin{gathered}
A=\left(\begin{array}{cccc}
0 & 2 \tau & \sqrt{\tau} & -\tau \sqrt{\tau} \\
-2 \tau \sqrt{\tau^{2}-\tau} & 0 & \sqrt{\tau^{2}-\tau} & \tau \sqrt{\tau^{2}-\tau} \\
-2 \tau \sqrt{\tau-1} & 2 \tau & \tau(\sqrt{\tau}+\sqrt{\tau-1}) & -\tau^{2}(\sqrt{\tau}-\sqrt{\tau-1}) \\
2 \sqrt{\tau-1} & 2 & \sqrt{\tau}-\sqrt{\tau-1} & -\tau(\sqrt{\tau}+\sqrt{\tau-1})
\end{array}\right), \\
B=\left(\begin{array}{cccc}
-2 \tau & 0 & \sqrt{\tau} & \tau \sqrt{\tau} \\
0 & 0 & i \sqrt{\tau}(\sqrt{\tau}-1) & -i \tau \sqrt{\tau}(\sqrt{\tau}-1) \\
-2 \tau(\tau+1-\sqrt{\tau}) & -2 i \tau(\sqrt{\tau}-1) & \tau & \tau^{2} \\
-2(\tau+1-\sqrt{\tau}) & 2 i(\sqrt{\tau}-1) & 1 & \tau
\end{array}\right) .
\end{gathered}
$$

On the other hand, to pass from the curve defined in $\mathbb{P}^{3}$ by $x^{2}=t z$ and $y^{2}=x t-(1+\tau) t z+\tau x z$ to the isomorphic curve defined by $x^{2}=t z$ and $y^{2}=$ $(t-z)\left(t-\left(2 \tau-2 \sqrt{\tau^{2}-\tau}-1\right)^{2} z\right)$ we can use the transformation of $\mathbb{P}^{3}$ induced by the matrix

$$
C_{2}=\left(\begin{array}{cccc}
-2 \tau & 0 & 1 & \tau \\
0 & -4 \sqrt{\tau(\tau-1) \xi} & 0 & 0 \\
2 \tau-2 \sqrt{\tau(\tau-1)} & 0 & -\xi & -\tau \\
2 \frac{-\sqrt{\tau(\tau-1)}+\tau}{\xi} & 0 & -\xi^{-1} & -\tau
\end{array}\right),
$$

where $\xi=2 \tau-2 \sqrt{\tau^{2}-\tau}-1$. Similarly, to pass to the third and fourth equations we 
can use the transformations induced by

and

$$
C_{3}=\left(\begin{array}{cccc}
0 & 0 & i & -i \tau \\
0 & 4 \frac{i \sqrt{\tau}}{1+\sqrt{\tau}} & 0 & 0 \\
2 \sqrt{\tau} & 0 & -1 & -\tau \\
2 \sqrt{\tau} & 0 & 1 & \tau
\end{array}\right)
$$

$$
C_{4}=\left(\begin{array}{cccc}
-1 & 0 & 1 / 2 & \tau / 2 \\
0 & 2 \frac{\sqrt{\tau-1}}{\sqrt{\tau}} & 0 & 0 \\
-i \sqrt{\tau} & 0 & -\frac{\sqrt{\tau-1}-i}{2 \sqrt{\tau}} & \frac{(\sqrt{\tau-1}+i) \sqrt{\tau}}{2} \\
i \sqrt{\tau} & 0 & -\frac{\sqrt{\tau-1}+i}{2 \sqrt{\tau}} & \frac{\sqrt{\tau}(\sqrt{\tau-1}-i)}{2}
\end{array}\right) .
$$

The coefficients for the hyperplanes in Table 1 are the images of the hyperplane defined by $a x+b y+c t+d z$ under the transformations $C_{2} A, C_{3} B$ and $C_{4} A B$.

It remains to prove the assertion about the correspondence. For this we first recall how one obtains an affine equation from the data in Table 1 . We first embed the genus 1 curve in $\mathbb{P}^{3}$ via $(x, y) \longmapsto\left[x, y, x^{2}, 1\right]$. If the coefficient $b_{k}$ of the hyperplane is different from 0 we make the change of variable $y=y-\left(1 / b_{k}\right)\left(a_{k} x+c_{k} t+d_{k} z\right)$ and then replace $t$ by $x^{2}, y$ by $y^{2}$ and $z$ by 1 to obtain an affine equation of the genus 3 curve in $\mathbb{P}^{2}$. If the coefficient $b_{k}$ is 0 then the points of intersection of the embedded curve and the hyperplane are stable under $y \longmapsto-y$. Hence we can, by means of a Möbius transformation, make a change of variables on the original equation and send these points to the point over 0 and $\infty$. An affine equation is then obtained by taking the standard hyperelliptic double cover.

Now we note that in all cases we in fact have $b=0$; see (5.2). This means that in all cases we will have $a_{2}=0, a_{3}=0$ and $a_{4}=0$ (this is in fact the reason for our choice of equations for the genus 1 curve). For the affine equation we have just constructed this means that $x$ only appears with even coefficients in the non-hyperelliptic case or is stable under $x \longmapsto 1 / x$ in the hyperelliptic case. In other words this means that the curve admits, in addition to the automorphism $(x, y) \longmapsto(x,-y)$ (respectively $(x, y) \longmapsto(-x, y)$ in the hyperelliptic case), the automorphisms $(x, y) \longmapsto(-x, y)$ and $(x, y) \longmapsto(-x,-y)$ (respectively the automorphisms induced by $x \longmapsto-1 / x$ and $x \longmapsto 1 / x)$. It is readily checked that these are the automorphisms $\psi_{1}$ and $\psi_{2}$ of Lemma 4.1.

The embedding in $\mathbb{P}^{3}$ of the vertices of the hexagon yields the following projective coordinates:

(1) $[0,0,1,0]$ for $p$;

(2) $[0,0,0,1]$ for $q$;

(3) $[1,0,1,1]$ (respectively $\left[1 / \lambda, \sqrt{(1 / \lambda)(1 / \lambda-1)(1 / \lambda-\tau)},(1 / \lambda)^{2}, 1\right]$ in case (iii)) for $r$

(4) $\left[\lambda, \sqrt{\lambda(\lambda-1)(\lambda-\tau)}, \lambda^{2}, 1\right]$ (respectively $\left.[1,0,1,1]\right)$ for $s$;

(5) $\left[\mu, \sqrt{\mu(\mu-1)(\mu-\tau)}, \mu^{2}, 1\right]$ (respectively $\left.\left[\tau, 0, \tau^{2}, 1\right]\right)$ for $t$. 
If we apply to these the matrices $C_{2} A, C_{3} B$ and $C_{4} A B$ it is then a simple, although tedious, matter to check for which images the $t$-coordinate or the $z$-coordinate is 0 , or in other words which, in the final equation, is fixed under $\psi_{1}$ and which is fixed under $\psi_{2}$. The identification of cases follows from this and Lemma 4.1.

REMARK 5.2. (1) We have used the convention that the modular invariants $\lambda, \mu$ and $v$ of the hexagon are such that $1<\lambda<\mu<v$. Recalling that the resulting genus 3 curve is hyperelliptic if and only if the coefficient $b_{k}$ is 0 , we see, comparing (5.2) and Table 1, that in cases (2.i)-(4.iii) in Subsection 4.3 the curve can be hyperelliptic only in case (2.i) if $v(\mu-\lambda)-\lambda(\mu-1)=0$, in case (3.iii) if $v=\lambda \mu$ and in case (4.iii) if $v(\lambda-1)-\lambda(\mu-1)=0$. We will see in Section 7 the geometric meaning of these conditions.

(2) If we follow the method indicated in the proof of Proposition 5.1 we will obtain equations with coefficients involving, in addition to $\lambda, \mu$ and $v$, terms in $\sqrt{\tau}$ and $\sqrt{\tau-1}$. The presence of these radicals is somewhat artificial since we can in each case remove them by means of suitable changes of variables. We do not give the details here since the resulting equations tend to be rather long in their general form, but the fact is that in all cases the genus 3 curves have equations with coefficients that are rational (and even polynomial) in $\lambda, \mu$ and $v$.

\section{An example}

The method we have described in the preceding section can easily be rendered totally automatic and can be used for extensive tests. We give here an example that we find rather striking.

Let $H$ be the rectangular hexagon with side lengths (in that order)

$$
\begin{aligned}
& l_{1}=\operatorname{arccosh}(\sqrt{10}), \quad \hat{l}_{3}=\operatorname{arccosh}\left(\frac{\sqrt{6}}{2}\right), \quad l_{2}=\operatorname{arccosh}(4), \\
& \hat{l}_{1}=\operatorname{arccosh}(2), \quad l_{3}=\operatorname{arccosh}\left(\frac{\sqrt{10}}{2}\right), \quad \hat{l}_{2}=\operatorname{arccosh}(\sqrt{6})
\end{aligned}
$$

(such a rectangular hexagon exists as one can check using the formulae in [2]).

We start by computing the modular invariants. The first set $\lambda_{1}, \mu_{1}$ and $v_{1}$ is obtained by sending the side with length $l_{1}$ onto $[0,1]$ and the side with length $\hat{l}_{2}$ onto $[\infty, 0]$. We then set for $i$ from 1 to 5

$$
\begin{aligned}
\lambda_{i+1} & =\frac{\lambda_{i}}{\left(\lambda_{i}-1\right)} \frac{\left(\mu_{i}-1\right)}{\mu_{i}}, \\
\mu_{i+1} & =\frac{\lambda_{i}}{\left(\lambda_{i}-1\right)} \frac{\left(v_{i}-1\right)}{v_{i}}, \\
v_{i+1} & =\frac{\lambda_{i}}{\left(\lambda_{i}-1\right)} .
\end{aligned}
$$

From a geometric point of view this corresponds to performing a cyclic permutation of the sides of the hexagon. For example the second set $\lambda_{2}, \mu_{2}$ and $v_{2}$ is obtained by sending the side with length $\hat{l}_{3}$ onto $[0,1]$ and the side with length $l_{1}$ onto $[\infty, 0]$.

We can also formulate this in terms of lengths of geodesic arcs in Figure 5. For example $\left(\lambda_{1}, \mu_{1}, v_{1}\right)$ are the invariants we will use when the geodesic arc between $p$ and $q$ in Figure 5 has length $\hat{l}_{2}$ and that between $q$ and $r$ has length $l_{1}$; the invariants $\left(\lambda_{2}, \mu_{2}, v_{2}\right)$ are the ones we will use when the length between $p$ and $q$ is $l_{1}$ and that between $q$ and $r$ is $\hat{l}_{3}$ and so forth by cyclic permutation. 
Numerically, we find that

$\lambda_{1} \sim 1.01857603000028, \quad \mu_{1} \sim 2.66666666666667, \quad v_{1} \sim 6.98142396999972$.

If we apply the method of the preceding section to this data (and also the data we obtain for rotations of the hexagon) we find for example that equation (1.i) for $\lambda_{3}$, $\mu_{3}$ and $\nu_{3}$ is approximately

$$
\begin{aligned}
y^{2}-1.0 x^{8}+11.666666666667 x^{6}-39.111111111111 x^{4} \\
+47.407407407407 x^{2}-18.962962962963,
\end{aligned}
$$

equation (1.ii) for $\lambda_{2}, \mu_{2}$ and $v_{2}$ is

$y^{2}-1.0 x^{8}+3.600000000000 x^{6}-3.200000000000 x^{4}-1.0 x^{2}+1.600000000000$,

equation (1.iii) for $\lambda_{3}, \mu_{3}$ and $v_{3}$ is

$$
\begin{aligned}
y^{2}-1.0 x^{8}-2.333333333333 x^{6}+3.333333333333 x^{4} \\
+4.629629629630 x^{2}-4.629629629630,
\end{aligned}
$$

equation (2.ii) for $\lambda_{6}, \mu_{6}$ and $v_{6}$ is

$$
\begin{aligned}
\left(y^{2}+2.200000000000 x^{2}-0.440000000000\right)^{2} & \\
- & 1.0 x^{4}+1.200000000000 x^{2}-0.200000000000,
\end{aligned}
$$

and equation (4.ii) for $\lambda_{1}, \mu_{1}$ and $v_{1}$ is

$$
\left(y^{2}-4.000000000000 x^{2}-0.875000000000\right)^{2}-1.0 x^{4}+0.500000000000 x^{2}-1.0 .
$$

These computations strongly suggest that we in fact have,

$$
\lambda_{1}=4-\frac{4}{3} \sqrt{5}, \quad \mu_{1}=\frac{8}{3}, \quad v_{1}=4+\frac{4}{3} \sqrt{5} .
$$

Using these values we can find simple expressions for other transforms.

\section{Special families}

In [3] P. Buser and R. Silhol exhibited one-parameter families of curves which are algebraic with respect to the natural projective structure of the moduli space and which at the same time are defined by algebraic relations between the FenchelNielsen coordinates of the associated hyperbolic surface. In the formulation given in Section 1 we can rephrase the last part of the above statement by stating that the families are obtained from pentagons with algebraic relations between the side lengths or the cosh of these lengths.

This type of construction can be generalized and we can construct such families in genus 3. We will limit here our description to a few such examples depending on one or two parameters.

We will need the following notations. We will call $l_{1}$ the length of the geodesic arc between $q$ and $r$ in Figure 5, $l_{2}$ the length of the geodesic arc between $s$ and $t$, $l_{3}$ the length between $u$ and $p, \hat{l}_{1}$ the length between $t$ and $u, \hat{l}_{2}$ the length between $p$ and $q$ and finally $\hat{l}_{3}$ the length between $r$ and $s$. We will also denote $L_{i}=\cosh \left(l_{i}\right)$ and $\hat{L}_{i}=\cosh \left(\hat{l}_{i}\right)$. Note that the hexagon is completely determined by the lengths $l_{1}, l_{2}$ and $l_{3}$, and that hence the hyperbolic structure of the surface is completely determined by these three lengths and the identification pattern, that is, the type (one of (1.i)-(4.iii)). 
We will also use the following convention; we will call $\lambda, \mu$ and $v$ the modular invariants of the hexagon obtained by mapping $l_{1}$ onto $[0,1]$ and $\hat{l}_{2}$ onto $[\infty, 0]$.

7.1.

Our first special family comes from hexagons for which $l_{1}=l_{3}$. Since the hexagon is then symmetric, the set $\{0,1, \lambda, \mu, v, \infty\}$ must be globally stable under the transformation $x \longmapsto v / x$ or in other words we must have $v=\lambda \mu$. Conversely if $v=\lambda \mu$ then the hexagon must be symmetric and satisfy $l_{1}=l_{3}$.

By Remark 5.2(1) the curve (3.iii) will be in this case hyperelliptic. Among the other equations obtained, one of interest is (4.iii), which is of the form $\left(y^{2}+\alpha x^{2}+\right.$ $\alpha)^{2}-x^{4}+\beta x^{2}-1$ (provided that $\mu \neq 1 /(2-\lambda)$; see Subsection 7.5 for the geometric meaning of this condition), where

$$
\alpha=\frac{\lambda \mu-2 \lambda+1}{\lambda \mu-2 \mu+1}, \quad \beta=2 \frac{\mu-2 \lambda}{\mu} .
$$

We can apply this to all the known values of $\lambda$ and $\mu$ that one can deduce from the computations made in [3]; this gives many exact examples.

\section{2 .}

For exactly the same reasons as in Subsection 7.1 the condition $v(\mu-\lambda)=\lambda(\mu-1)$ will be satisfied if and only if $l_{2}=l_{3}$. In this case the curve (2.i) will be hyperelliptic. Among the other equations one of interest this time is (4.i), which is of the form $\left(y^{2}+\alpha x^{2}-\alpha\right)^{2}-x^{4}+\beta x^{2}-1$, where

$$
\alpha=\frac{\lambda-2 \mu+\mu^{2}}{\lambda-\mu^{2}}, \quad \beta=2 \frac{\lambda-2}{\lambda} .
$$

Again we can apply this to the computations in [3] to get new exact examples.

\section{3 .}

Our third family will be defined by the condition $l_{1}=\hat{l}_{1}$. This condition implies that we also have $l_{2}=\hat{l}_{2}$ and $l_{3}=\hat{l}_{3}$ and hence that the hexagon admits a central symmetry. From this we can conclude that $v /(\mu-1)=\lambda /(\lambda-1)$. In this case, and only in this case, the curve (4.iii) will be hyperelliptic.

We also have one-parameter families. To construct such families one can start with the one-parameter families of hexagons described in [3] and construct the corresponding genus 3 families. We do this for two of these.

\section{4}

Consider hexagons for which $l_{1}=l_{2}=l_{3}$. Then we are at the intersection of the families in Subsections 7.1 and 7.2. This implies that the modular invariants are of the form

$$
\lambda=\frac{\mu^{2}-\mu+1}{\mu}, \quad \mu, \quad v=\mu^{2}-\mu+1 .
$$


We can use these values to simplify equations (4.i) and (4.iii). For (4.iii) we can simplify even further. Writing the equation in homogeneous form we find after an obvious change of variables (replacing $x$ by

$$
x \sqrt{\mu(\mu-2) /(2(\mu-1))},
$$

$z$ by

$$
z \sqrt{\mu(\mu-2) /(2(\mu-1))}
$$

and $y$ by $i y)$

$$
x^{4}+y^{4}+z^{4}+\frac{\mu^{2}-2 \mu+2}{\mu-1}\left(x^{2} y^{2}+y^{2} z^{2}+z^{2} x^{2}\right)
$$

(provided that $\mu \neq 2$, see below). Similarly we find, for equation (3.i),

$$
x^{4}+y^{4}+z^{4}-\frac{\mu^{2}+1}{\mu}\left(x^{2} y^{2}+y^{2} z^{2}+z^{2} x^{2}\right) .
$$

Both these equations exhibit a group of automorphisms containing the permutation group $\Xi_{4}$ (in other words these curves are in the KFT family of [9]).

For $L_{1}=L_{2}=L_{3}=2$, the hexagon is the symmetric hexagon, and it is easy to find that $\mu=2$ in this case. The curve (4.iii) will in fact be hyperelliptic and the equation is $y^{2}-x^{8}-14 x^{4}-1$, which is known to be the only hyperelliptic curve in the KFT family (see [9]). For (3.i) we find

$$
x^{4}+y^{4}+z^{4}-5 / 2\left(x^{2} y^{2}+y^{2} z^{2}+z^{2} x^{2}\right) .
$$

\section{5}

The last family we will consider is the one for which we have both $l_{1}=l_{3}$ and $l_{1}=\hat{l}_{1}$. The conditions we have found in Subsections 7.1 and 7.3 show that we must have $\mu=1 /(2-\lambda)$ and $v=\lambda /(2-\lambda)$. The curve (3.iii) will be hyperelliptic and its equation in terms of $\lambda$ can be easily expressed. Applying the method of Section 5 we find an equation of the form $y^{2}-\left(x^{2}-a\right)\left(x^{2}-1 / a\right)\left(x^{2}+a\right)\left(x^{2}+1 / a\right)$ with

$$
a=\frac{i(\lambda-1)-1+\sqrt{(2-\lambda) \lambda}}{i(\lambda-1)+1-\sqrt{(2-\lambda) \lambda}}
$$

Expanding the equation yields

$$
y^{2}-x^{8}-\left(4 \lambda^{2}-8 \lambda+2\right) x^{4}-1 .
$$

The curve (4.iii) will also be hyperelliptic and similarly the equation can be expressed as

$$
y^{2}-x^{8}+2 \frac{\lambda^{2}-2 \lambda-1}{(\lambda-1)^{2}} x^{4}-1 .
$$

Another curve of interest in this case will be (2.iii) which is related to (3.iii). We find, after homogenizing and substituting $z \sqrt{\lambda /(2-\lambda)}$ for $z$, that the equation is

$$
y^{4}-x^{4}+2 \frac{\lambda^{2}-2 \lambda+2}{\lambda(2-\lambda)} x^{2} z^{2}-z^{4},
$$

but we can make a further change of variables; we can replace $x$ by $(\sqrt[4]{\lambda(2-\lambda)} / \sqrt{2})$ 
$(x+i z)$ and $z$ by $(\sqrt[4]{\lambda(2-\lambda)} / \sqrt{2})(i x+z)$, which yields

$$
y^{4}-x^{4}-\left(4 \lambda^{2}-8 \lambda+2\right) x^{2} z^{2}-z^{4}
$$

(compare with equation (7.1)).

Using the computations in [3] for the corresponding hexagons we find, among others, for curves (3.iii) and (2.iii), the following:

(1) For $L_{1}=L_{3}=\sqrt{2+\sqrt{2}}$ and $L_{2}=1+\sqrt{2}$ (for which we have $\lambda=1+\sqrt{2} / 2$, $\mu=2+\sqrt{2}$ and $v=3+2 \sqrt{2}$ ),

$$
y^{2}-x^{8}-1 \text { and } y^{4}-x^{4}-z^{4} .
$$

(2) For $L_{1}=L_{2}=L_{3}=2$ (for which we have $\lambda=3 / 2, \mu=2$ and $v=3$ ),

$$
y^{2}-x^{8}+x^{4}-1 \text { and } y^{4}-x^{4}+x^{2} z^{2}-z^{4} .
$$

The exact values for the modular invariants of many other hexagons satisfying the above conditions can be deduced from the computations in [3], and from these we can get other exact examples.

\section{References}

1. A. Aigon, 'Transformations hyperboliques et courbes algébriques en genre 2 et 3', Thèse, Université Montpellier 2, 2001

2. P. BusER, Geometry and spectra of compact Riemann surfaces (Birkhäuser, Boston, 1992).

3. P. Buser and R. Silhol, 'Geodesics, periods and equations of real hyperelliptic curves', Duke Math J. 108 (2001) 211-250.

4. H. FARKAS and I. KRA, Riemann surfaces, Graduate Texts in Mathematics 71 (Springer, Berlin, 1980).

5. P. Gianni, M. Seppälä, R. Silhol and B. Trager, 'Riemann surfaces, plane algebraic curves and their period matrices', J. Symbolic Comput. 26 (1998) 789-803.

6. P. Henrici, Applied and computational complex analysis - Vol. 3 (John Wiley, New York, 1986)

7. B. Maskit, 'Matrices for Fenchel-Nielsen parameters in genus 2', Contemp. Math. 240 (1999) 259-267.

8. Z. Nehari, Conformal mapping (McGraw-Hill, New York, 1952).

9. R. Rodríguez and V. GonzÁlez-Aguilera, 'Fermat's quartic curve, Klein's curve and the tetrahedron', Contemp. Math. 201 (1997) 43-62.

10. R. Silhol, 'Hyperbolic lego and algebraic curves in genus 2 and 3', Contemp. Math., to appear.

11. J. H. Silverman, The arithmetic of elliptic curves, Graduate Texts in Mathematics 106 (Springer Berlin, 1986)

Département de Mathématiques

\section{EPFL}

CH-1015 Lausanne

Switzerland

aline.aigon@epfl.ch
Département de Mathématiques,

UMR 5030

Université Montpellier II

Place E Bataillon

34095 Montpellier Cedex 5

France

rs@math.univ-montp2.fr 